\title{
VARIACIÓN DE LA VISCOSIDAD PLASMÁTICA EN CONEJOS INDUCIDOS A UNA DIABETES ALOXÁNICA
}

\section{VARIATION OF PLASMATIC VISCOSITY IN ALLOXAN-INDUCED DIABETIC RABBITS}

\author{
Gonzales Medrano, M. Fernando ${ }^{a}$, Infantas Mesías, Delia ${ }^{a}$ y Sam Torres, Rosa ${ }^{b}$
}

\section{RESUMEN}

Se investigó la variación de la viscosidad plasmática en conejos inducidos a una diabetes aloxánica. Adicionalmente, los valores de hematocrito, densidad plasmática y deformabilidad celular de glóbulos rojos fueron estimados. Los animales diabéticos presentaron pérdida de peso mientras que la densidad y viscosidad plasmática se incrementaron. Los valores de hematocrito no mostraron diferencias estadísticas significativas. Estos resultados sugieren que el incremento del valor de la viscosidad plasmática es un factor involucrado en la viscosidad sanguínea descrita para pacientes diabéticos con descompensación.

Palabras clave: Viscosidad plasmática, diabetes aloxánica, conejos.

\begin{abstract}
The variation of plasmatic viscosity in alloxan-induced diabetic rabbits was investigated. Additionally, hematocrit values, plasmatic density and red blood cell deformability were estimated. The diabetic animals showed loss weight whereas the density and viscosity of plasma were increased. The Hematocrit values did not show any significative statistical differences. These results suggest the increased level of plasmatic viscosity is an involucrated factor in the blood viscosity described for decompensated diabetic patients.
\end{abstract}

Key Words: Plasmatic viscosity, alloxan-diabetes, rabbits.

\section{INTRODUCCIÓN}

La diabetes mellitus es un síndrome que manifiesta un trastorno metabólico relacionado a una hiperglucemia, consecuencia de una deficiencia en la secreción de insulina o en el efecto biológico de la misma, la cual daña muchos de los sistemas del cuerpo [1]. Desde el punto de vista epidemiológico, este trastorno metabólico se ha convertido en una enfermedad muy preocupante para la Organización Mundial de la Salud (OMS) debido a que se observa un fuerte crecimiento de personas que la padecen. Se calcula actualmente más de 170 millones de personas que sufren de diabetes en el mundo, y se prevé que este número se habrá duplicado para el año 2030 [2].

Los conejos han demostrado ser un buen modelo biológico para estudios experimentales con diabetes, debido a una respuesta muy similar a los humanos, aún más que en otros animales [3]. Las alteraciones existentes en animales con diabetes pueden ser muy graves e incluso fatales. La forma más común de monitorear este síndrome, es a través de la cantidad de glucosa en la sangre. Para cumplir nuestros objetivos, se realizarán mediciones de la viscosidad plasmática para determinar si existe una relación entre su resistencia al flujo y la diabetes.

Debido a que el grado de viscosidad sanguínea está determinado por la viscosidad plasmática, hematocrito, deformabilidad y agregación eritrocitaria [4], donde los parámetros más relacionados directamente son la viscosidad plasmática y el hematocrito. Además, han

\footnotetext{
${ }^{a}$ Laboratorio de Fitoquímica, Universidad Nacional Agraria La Molina.

${ }^{\mathrm{b}}$ Laboratorio de Microbiología y Parasitología, Universidad Nacional Mayor de San Marcos.
}

sido descritas alteraciones en la microcirculación, mediante un daño vascular preferentemente en la membrana basal de los pequeños vasos, aumentando la producción de algunas glicoproteínas que pueden provocar engrosamiento secundario de la membrana basal. Por lo que la adherencia plaquetaria puede ser la causa de un aumento en la viscosidad plasmática [5]. Con la finalidad de determinar el causante de las variaciones en la viscosidad sanguínea descrita en conejos químicamente diabéticos, se hará uso de la medición de la viscosidad plasmática, hematocrito y un estudio de presencia o ausencia de deformabilidad celular.

Otros autores evaluaron la variación de la viscosidad sanguínea y plasmática en relación al grado de compensación metabólica en diabéticos, encontrando una notable viscosidad sanguínea en pacientes diabéticos no compensados sugiriendo su relación con ciertas propiedades de los glóbulos rojos como la flexibilidad de su membrana y por tanto su deformabilidad [6].

En el presente trabajo de investigación se planteó como objetivo general determinar la existencia de una variación de viscosidad plasmática en relación a la diabetes. Adicionalmente, la inducción de un cuadro de diabetes aloxánica estable en conejos, la evaluación de algunos parámetros reológicos sanguíneos en conejos normoglicémicos y conejos químicamente diabéticos y la evaluación del grado de relación de tres variables descritas por la literatura se ven alteradas en condiciones diabéticas fueron analizadas. 


\section{MATERIALES Y MÉTODOS}

\section{Material Biológico}

Se utilizó 7 conejos de raza Nueva Zelanda línea pura de ambos sexos previamente aclimatados, de 59 días y pesos entre los $1,995 \mathrm{Kg}$ a $2,150 \mathrm{Kg}$. Se agruparon aleatoriamente y distribuidos al azar e 2 grupos. Se mantuvieron a condiciones naturales de humedad, temperatura con promedio de $27^{\circ} \mathrm{C}$ e iluminación de 12 horas de luz y 12 horas de oscuridad aproximadamente. Recibieron una dieta diaria de $150 \mathrm{~g}$ de alimento balanceado para conejos y agua ad libitum. Estudios previos no encontraron diferencias significativas entre animales machos y hembras en relación con la incidencia, evolución y severidad del síndrome diabético [1]. Se asumieron las demás variables constantes.

\section{Obtención de Muestras de Sangre}

El manejo de los animales de laboratorio fue realizado bajo los lineamientos de bioética y recomendaciones para su utilización [7]. Los animales fueron sometidos a un ayuno previo de 20-21 horas y pesados en una balanza gravimétrica KERN 440-35N. $14 \mathrm{ml}$ de sangre fueron extraídos a través de la vena marginal de la oreja exaltada con xilol, posteriormente mezclada con anticoagulante EDTA a $1-2 \mathrm{mg} / \mathrm{ml}$. Se separó el plasma sanguíneo por centrifugación a 3500 rpm por 35 minutos en una centrífuga Hettich Universal II y llevado a $4^{\circ} \mathrm{C}$ hasta su posterior utilización. Los valores de hematocrito fueron expresados en valor de porcentaje (\%). Se realizó hemostasia en la zona de corte para evitar hemorragias.

\section{Inducción de diabetes}

Se utilizó el aloxano como reactivo inductor de la diabetes por su capacidad necrótica sobre las células de los islotes de Langerhans $(8,9)$. Se calculó la cantidad de reactivo necesario para la inducción de diabetes a una concentración final de $120 \mathrm{mg} / \mathrm{ml}$. Se inyectó la solución de aloxano disuelto en $1 \mathrm{~mL}$ de suero fisiológico para mamíferos estéril $(\mathrm{NaCl}$ al $0,98 \%)$ por vía endovenosa (10). Se evaluó su condición antes de la inducción y 7 días después de la inducción diabética utilizando tiras de glucosa en un glucómetro comercial. La condición diabética fue considerada como tal para conejos con concentraciones de glucemia en sangre mayores que $250 \mathrm{mg} / \mathrm{dL}$.

\section{Medición de la viscosidad plasmática}

Se determinó la viscosidad plasmática utilizando un viscosímetro capilar de Ostwald, medición que obedece a la ecuación expresada por Poiseuille (11). La medición de los valores se realizó en un baño maría a temperatura controlada de $39^{\circ} \mathrm{C}$ con muestras y viscosímetro previamente atemperadas por 10 minutos. Se midió el tiempo de flujo con 10 repeticiones y el valor fue determinado por el promedio aritmético de estos. Se determinó las densidades a condiciones controladas, expresándolas en $\mathrm{Kg} / \mathrm{m}^{3}$.

La calibración del viscosímetro de Ostwald fue realizada utilizando los datos teóricos de la viscosidad y densidad del agua a $39{ }^{\circ} \mathrm{C}$, midiendo el tiempo de flujo de una muestra de agua bidestilada estéril. Los demás valores fueron considerados invariables, obteniéndose una constante de calibración para el viscosímetro a $39^{\circ} \mathrm{C}$.

\section{Frotis sanguíneo y observación celular}

Muestras frescas de sangre fueron utilizadas para realizar el frotis sanguíneo de los animales de experimentación Se siguió el procedimiento descrito por Voigt (12). Los frotis, previamente secados, fueron llevados a un estereoscopio Nikon eclipse E200 para su evaluación y detectar posibles anomalías.

\section{RESULTADOS}

Los conejos adquirieron una diabetes aloxánica estable. En la tabla 1 se muestran los valores obtenidos para la medición de glucosa sanguínea como consecuencia de la inducción de diabetes. Consistentes variaciones se encontraron para los indicadores de ganancia de peso (figura $1 \mathrm{~A}$ ) y valores de densidad plasmática (figura 1C), demostrando diferencias significativas con una probabilidad del $5 \%$ utilizando la prueba estadística no paramétrica de Mann-Whitney para 2 muestras independientes. Contrariamente, no se obtuvieron diferencias significativas entre las medias de los valores de volumen celular aglomerado de ambos grupos (figura 1B).

Tabla 1: datos obtenidos al inicio (1) y 7 días después de la inducción de diabetes (2)

\begin{tabular}{|l|l|l|l|l|l|l|l|}
\hline Grupo & Conejo & Peso 1 & Peso 2 & Glu 1 & Glu 2 & Hcto 1 & Hcto 2 \\
\hline Control & A & 2,090 & 2,620 & 80 & 105 & 46,428 & 50,000 \\
\hline Control & B & 1,995 & 2,500 & 84 & 109 & 46,428 & 45,710 \\
\hline Control & C & 2,150 & 2,635 & 75 & 111 & 45,714 & 43,570 \\
\hline Diabetes & D & 2,005 & 2,220 & 84 & 498 & 44,643 & 44,286 \\
\hline Diabetes & E & 2,090 & 2,405 & 93 & $*$ & 44,643 & $*$ \\
\hline Diabetes & F & 2,140 & 2,350 & 66 & 437 & 44,643 & 43,570 \\
\hline Diabetes & O & 2,140 & 2,350 & 108 & 327 & 46,428 & 44,286 \\
\hline
\end{tabular}

${ }^{*}$ No se pudo determinar los valores por muerte del animal.

La prueba estadística no paramétrica de Mann Whitney para 2 muestras independientes con una probabilidad del $13 \%$ mostró diferencias significativas entre las medias de los valores de viscosidad plasmáticas para el grupo control en contraste con el grupo diabético. Se utilizó la prueba estadística no paramétrica de Wilcoxon para 2 muestras relacionadas con una probabilidad del $11 \%$ para determinar la existencia de variación entre las medianas de la viscosidad plasmática en conejos antes y después de ser inducidos a una diabetes aloxánica. Los resultados indicaron diferencias significativas, confirmando un incremento de la viscosidad plasmática en conejos inducidos a una diabetes aloxánica (figura 1D). 


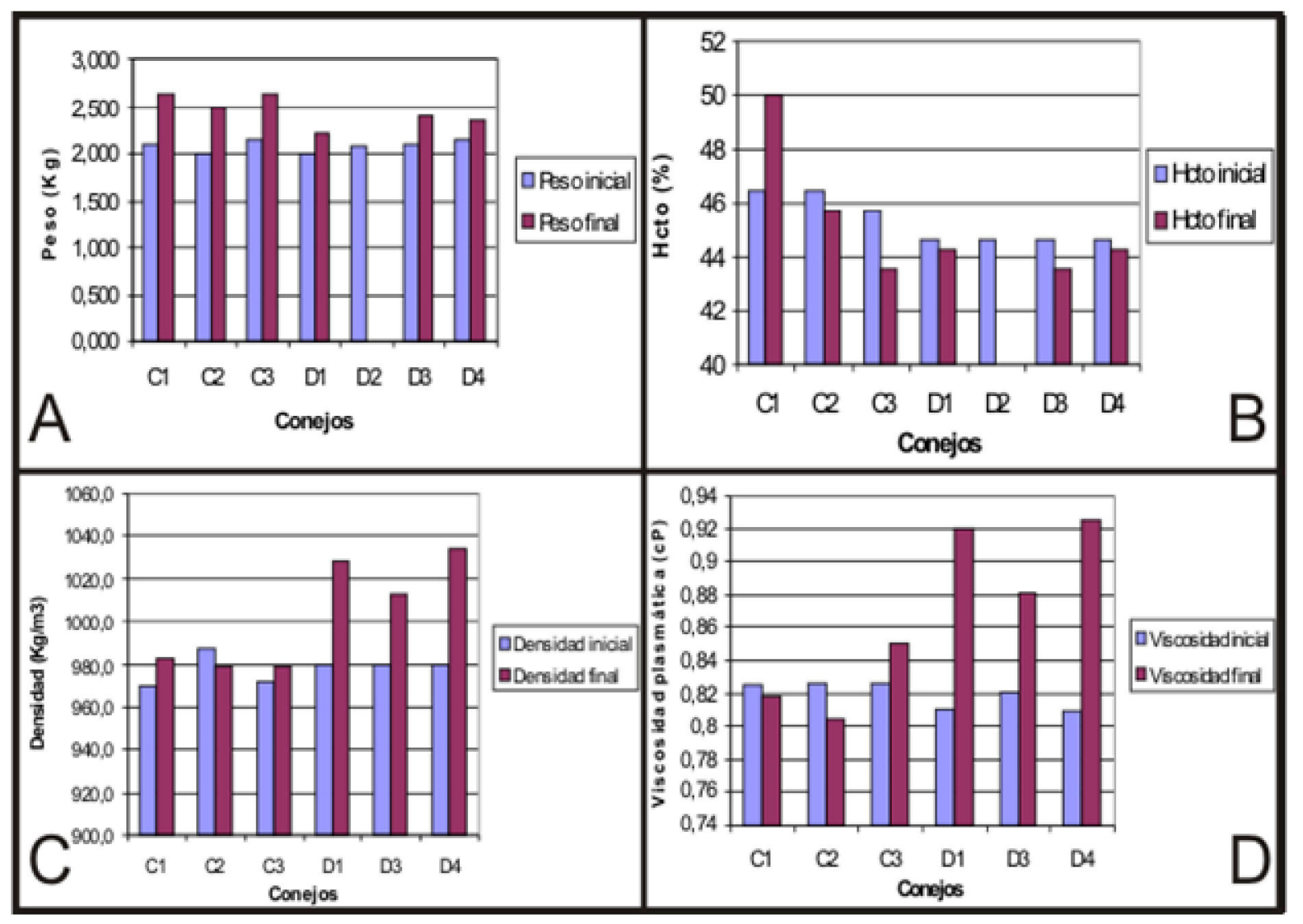

Figura 01: Comparación del peso $(A)$, hematocrito $(B)$, densidad $(C)$ y viscosidad $(D)$ antes y después de la inducción de la diabetes.

\section{DISCUSIONES}

En condiciones diabéticas los individuos no pueden hacer un uso eficiente de la glucosa sanguínea por falta de insulina, que es la responsable del paso de la glucosa al interior de la célula, en consecuencia eliminándola por la orina (glucosuria). Esta deficiencia promueve la movilización de las grasas y un exagerado catabolismo de los ácidos grasos con un aumento de cuerpos cetónicos y triglicéridos (13), explicándose así las diferencias en la ganancia de peso entre los 2 grupos.

El estrés puede ser un causante de un incremento de hematocrito por la liberación de células sanguíneas del bazo al torrente sanguíneo, sin embargo en casi todos los individuos se ha presentado una ligera disminución, aunque no significativa, la cual puede ser explicada debido a que todos los conejos fueron sometidos a una sangría de alrededor de un $12 \%$ de volumen sanguíneo total y esto puede ser causa de un ligero cuadro de anemia (12).

Se cree que la causa del incremento de la densidad plasmática en conejos diabéticos puede deberse a la producción de ciertas proteínas de bajo peso molecular, por lo que se recomienda realizar estudios posteriores orientándolos hacia estos parámetros.
Las variaciones en la viscosidad plasmática nos sugieren que esta se ve incrementada en condiciones diabéticas, donde la viscosidad sanguínea se ve alterada también por los valores plasmáticos. En contraste con otros autores quien sugiere esta variación en la viscosidad sanguínea se deben exclusivamente al componente hemático de la elasticidad de la membrana eritrocitaria (6). Aún se sigue evaluando esta controversia, debido a los resultados confrontados por otros autores. Estudios posteriores deberían direccionar las investigaciones a la evaluación de concentraciones proteicas en el plasma sanguíneo bajo estas condiciones.

Nosotros sugerimos que este incremento de la viscosidad plasmática puede ser causado por proteínas de bajo peso molecular, además de determinadas $\alpha$ glicoproteínas, $\beta$-glicoproteínas, fibrinógeno, proteínas del complemento entre otras descritas por estudios previos (6), las mismas que evidenciaron un aumento de densidad.

Valores de la concentración de glucosa tomados 33 días después de la inducción de diabetes mostraron una disminución considerable lo que puede ser causado por un reajuste metabólico del animal con tendencia a normalizarse. Sin embargo, la condición diabética fue mantenida, debido a que las concentraciones de glucosa 
que se midieron estuvieron dentro del rango diabético (262-337 mg/dL).

\section{CONCLUSIONES}

El viscosímetro capilar de Ostwald ha representado una buena herramienta para evidenciar diferencias significativas entre medidas de viscosidad plasmática para conejos sanos y diabéticos.

De los 3 factores que han sido evaluados (hematocrito, viscosidad plasmática y deformabilidad celular), los cuales influyen directamente sobre la variación de la viscosidad sanguínea, la viscosidad plasmática ha demostrado ser la más relacionada en condiciones de diabetes y una variable que influencia el aumento de la viscosidad sanguínea reportada en pacientes diabéticos no compensados (6).
El reactivo diabetogénico Aloxano ha demostrado ser un buen inductor de un cuadro diabético estable en conejos.

\section{AGRADECIMIENTOS}

Los autores agradecen a Juan Juscamaita Morales por su invaluable asesoría, a la Ing. Gloria Palacios Pinto por su apoyo en el manejo animal y al estudiante de Biología Joel Rosales Manta por su constante asistencia durante el desarrollo de la investigación

\section{REFERENCIAS BIBLIOGRÁFICAS}

[1]. Ramos H, Méndez J. Diabetes Mellitus experimental. Ciencia Veterinaria. 1994; 6(12): $347-377$

[2]. Organización Mundial de la Salud. 2005. Día Mundial de la Diabetes. Comunicado de prensa conjunto OMS/FID. www.who.int (acceso a la página: diciembre 12, 2007)

[3]. Fleitas A, Simón R, Almeida G, Quintela A, Alfonso M. Modelo Experimental de Diabetes en Conejos. Revista Cubana de Angiología y Cirugía Vascular. 2000; 1(1): 10-14

[4]. Reinhart W. Molecular biology and self-regulatory mechanism of blood viscosity: A review. Biorheology. 2001; 38(23): 203-212.

[5]. Farrel A. Acquired perforating dermatosis in renal and diabetic patients. The Lancet. 1997; 349: 985-986.

[6]. Pola P, Savi L, Serricchio M, Tondi P, Capaldi L. Variaciones de la viscosidad sanguínea y plasmática en relación al grado de compensación metabólica en la diabetes. Angiología. 1981; 33(3): 129-143.

[7]. Morton D, Abbot D, Barclay R, Close B, Ewbank R, et al. Renoval of blood from laboratory mammals and birds. Laboratory Animals. 1993; 27(1): 1:22.

[8]. Szkudelski T. The Mechanism of Alloxan and Streptoxotocin Action in B Cells of the Rat Páncreas. Physiological Reserch. 2001; 50(6): 536-546.

[9]. Rabinovitch A, Suárez-Pinzón W. Cytokines and Their Roles in Pancreatic Islet $\beta$-Cell Destruction and InsulinDependent Diabetes Mellitus. Biochemical Pharmacology. 1998; 55(8): 1139-1149.

[10]. García M. Influencia de la diabetes experimental en respuestas serotonérgicas a nivel cardiovascular y renal (Tesis Doctoral). Universidad de Salamanca, Facultad de Farmacia. 2000.

[11]. Maron S, Pruton C. Fundamentos de Fisicoquímica. Editorial Limusa. México. 1973. pp. 66-71.

[12]. Voigt G. Conceptos y técnica shematológicas para técnicos veterinarios. Edición española. Editorial ACRIBIA S.A. España. 2000. pp. 144.

[13]. Narayan A, Kumar R, Watal G. Hypoglycemic effect of Murraya koenigii on normal and alloxan-diabetic rabbits. Journal of Ethnopharmacology. 2005; 97: 247-251.

E-mail: fgonzalesm@gmail.com 